\title{
La práctica constructiva local: debilidad u oportunidad para una arquitectura sustentable
}

David Elsinger ${ }^{1}$; Beatriz Garzón ${ }^{2}$

\section{RESUMEN:}

Transitando el año 2018, la Práctica Constructiva (PC) urbano-residencial de la provincia de Tucuman escasamente construye alguna forma de arquitectura sustentable. Tanto la falta de conocimiento de los actores de la construcción y los consumidores de la misma así como la escasa promoción y regulación para aplicar construcción sustentable constituyen tal vez, algunos de los problemas que explican el estado actual de las ciudades de la provincia.

En el análisis de los diferentes actores involucrados en la PC, se identifican a seis: 1) Profesionales; 2) Constructores; 3) Gobierno local; 4) Universidad; 5) Comitentes y 6) Proveedores de materiales. El objetivo del trabajo es realizar un primer abordaje a los dos primeros actores, así como las relaciones que los vinculan.

La metodología combina e integra técnicas cualitativas: una encuesta realizada a arquitectos activos y la entrevista semi-estructurada a constructores con trayectoria en diversas especialidades. Otra técnica es el análisis documental de informes y normativas existentes.

Las conclusiones giran en torno a la comprensión de los actores intervinientes, su comportamiento e interrelaciones. Se procura encontrar los significados que cada grupo asigna al proceso constructivo para determinar acciones que materialicen una inserción eficiente de arquitectura sustentable en la práctica constructiva local.

Palabras clave: Práctica constructiva, arquitectura sustentable, constructores y arquitectos.

\section{ABSTRACT:}

During the year 2018, the residenceurban construction practice ( $P C$ ) of the state of Tucumán scarcely build any kind of sustainable architecture. Both the lack of

${ }^{1}$ CONICET, FAU-UNT. Av. Nestor Kirchner N¹900, CP4000, San Miguel de Tucumán, Tucumán, Argentina. Email: arq.delsinger@gmail.com

${ }^{2}$ CONICET, MinCyT, FAU-SCAIT, UNT. Av. Nestor Kirchner N¹900, CP4000, San Miguel de Tucumán, Tucumán, Argentina. 
information (of the builder's and owners) and the scar promotion and regulation to apply sustainable construction, perbaps explain the current state of the cities from Tucumán.

About the different players involve in the $P C$, we can identify six: 1. Professionals; 2. Builders; 3. Local government; 4. University; 5. Customers and 6. Material suppliers. The objective of this work it is to make the first approach to the Professionals and the Builders and their relationships.

The methodology combine and integrates qualitative techniques: a poll made to architects in activities and a non-structured interview was made to the experienced builders in diverse specialties. Another technique used was documental analysis of reports and currents normatives.

The conclusions are the comprehension of the selected players, their behavior and their relationships. We are looking to find the give-meaning that each group assigns to the construction process to determinate actions to make an efficient insertion of sustainable architecture in the local builder practice.

Keywords: builder practice, sustainable architecture, builders and architects.

\section{INTRODUCCIÓN:}

El presente trabajo forma parte del Proyecto de investigación titulado: Inserción de la arquitectura sustentable en la práctica constructiva urbana-residencial de Tucumán ${ }^{1}$. En la búsqueda de comprender la constitución y el funcionamiento de la práctica constructiva se toma en consideración el concepto de habitus de Bourdieu (1980) para una mejor comprensión de la práctica.

Expresa que: "Producto de la historia, el habitus origina prácticas, individuales y colectivas, y por ende historia, de acuerdo con los esquemas engendrados por la historia; es el habitus el que asegura la presencia activa de las experiencias pasadas que, registradas en cada organismo bajo la forma de esquemas de percepción, de pensamientos y de acción, tienden, con más seguridad que todas las reglas formales y todas las normas explícitas, a garantizar la conformidad de las prácticas y su constancia a través del tiempo". (Bourdieu, 1980: p.89) De esta manera, la PC implica la interacción de distintos actores (agentes) que se estructuran históricamente en un medio complejo, con el fin de la producción edilicia en particular y del hábitat en general.

Dentro de la complejidad de esta práctica es posible realizar una primera clasificación de seis actores que la constituyen: 1 ) Profesionales: en todas sus escalas, desde los grandes estudios de arquitectura hasta profesionales libres; 2) Constructores: contratistas y empleados de la construcción 3) Gobierno local: Código de edificación, Políticas y Gestión Gubernamental; 4) Universidad: Plan de estudio y perfil del egresado; 5) Comitentes: Privados y Públicos y 6) Proveedores de materiales.

${ }^{1}$ Perteneciente al Proyecto "Habitat Sustentable y Saludable"CONICET, FAU-SCAIT, UNT 
En consecuencia: ¿Cuál es el comportamiento de los Profesionales y los Constructores frente a la probable inserción de una Arquitectura Sustentable (AS) en nuestro medio? Responder esta pregunta es el objetivo principal del presente estudio.

\section{MATERIALES Y MÉTODOS:}

La metodología utilizada comprende dos entrevistas en profundidad semiestructuradas a contratistas del medio. E1 primero de ellos con su mayor campo de acción en San Miguel de Tucumán (capital), y el segundo en la ciudad de Concepción (72km al Sur de la capital). Por otro lado, se realizó una primera encuesta con muestra aleatoria a cuarenta y ocho (48) arquitectos activos de ambas ciudades ${ }^{2}$.

Las entrevistas en profundidad tuvieron como objetivo comprender las trayectorias de los entrevistados (comienzos laborales, formación, especialización, lugares de trabajo) y por otro lado sus impresiones en relación a su oficio, al manejo de sus empleados, la gestión de sus servicios y, con especial atención, a la relación con los arquitectos y los clientes.

La encuesta realiza una clasificación por edades para conocer la heterogeneidad etaria del grupo y, por consiguiente, su trayectoria. Se indaga sobre la autonomía o dependencia en las relaciones profesionales $y$, dentro de ellas, cuales son las actividades realizadas en la labor diaria. A partir de allí, se indaga sobre el grado de conocimiento en AS y las fuentes de formación para luego especificar si la eficiencia energética (EE) y la AS son un objetivo en su práctica profesional. Finalmente, se interroga sobre la factibilidad de incorporación de disposiciones obligatorias de AS en las normativas y códigos de edificación vigente y sobre las posibilidades de inserción de la AS en nuestro medio.

\section{RESULTADOS:}

Entrevistas: E1 entrevistado $n^{\circ} 1$, nace en Santiago del Estero en 1960 (58 años de edad) en el departamento Choya. Migra a Buenos Aires y comienza su actividad en la construcción como "ayudante" entre

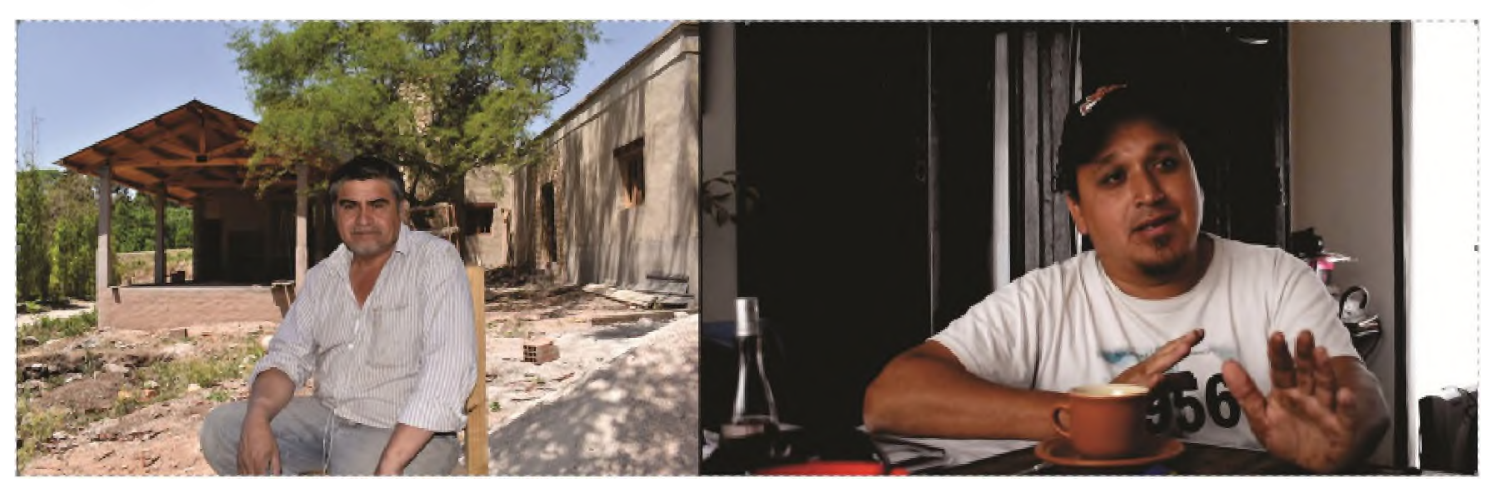

2 La muestra representa el 8,4\% de las 572 matrículas activas del Colegio de Arquitectos de Tucumán en el año 2018. Fuente: http://www.catonline.org.ar/ejercicioprofesional/matricula-profesional-activa 
1977 y $1983^{3}$. Aprende el oficio de albañil y "frentista". Viaja a Tucumán y ocupa la actividad de "oficial" en obras realizadas con familiares. En el año 1993 comienza sus actividades como contratista y en paralelo es contratado por una empresa constructora donde desarrolla obras de gran envergadura. Como contratista, declara la construcción de 35 edificaciones nuevas y 500 clientes con los que realizó trabajos de refacción y ampliación en diferentes localidades de Tucumán. Demuestra conocimientos claros en temas relacionados a construcciones térmicamente y acústicamente eficientes además de una amplia experiencia en la coordinación de los obreros y las relaciones con profesionales en obra.

El entrevistado ${ }^{\circ} 2$, nace en la ciudad de Concepción, Tucumán en 1981 (37 años de edad). Comienza sus trabajos alrededor de los 17 años cumpliendo la función de "ayudante" y aprende el oficio por parte de su padre y hermanos mayores que ya eran albañiles. Con ellos, reside por trabajo en Buenos Aires desde 2008 a 2010. Luego, es contratado por empresas y en paralelo comienza sus actividades como contratista. Manifiesta haber trabajado en diversas localidades de la provincia. Declara la construcción de más de 15 viviendas trabajando con su padre, 4 viviendas desde el 2010 como contratista y 50 a 60 clientes de refacción/ ampliación. Maneja conceptos y consideraciones térmicas relacionadas a la envolvente de una edificación.

Ambos entrevistados continúan trabajando como contratistas y se muestran predispuestos a asimilar cualquier cambio que

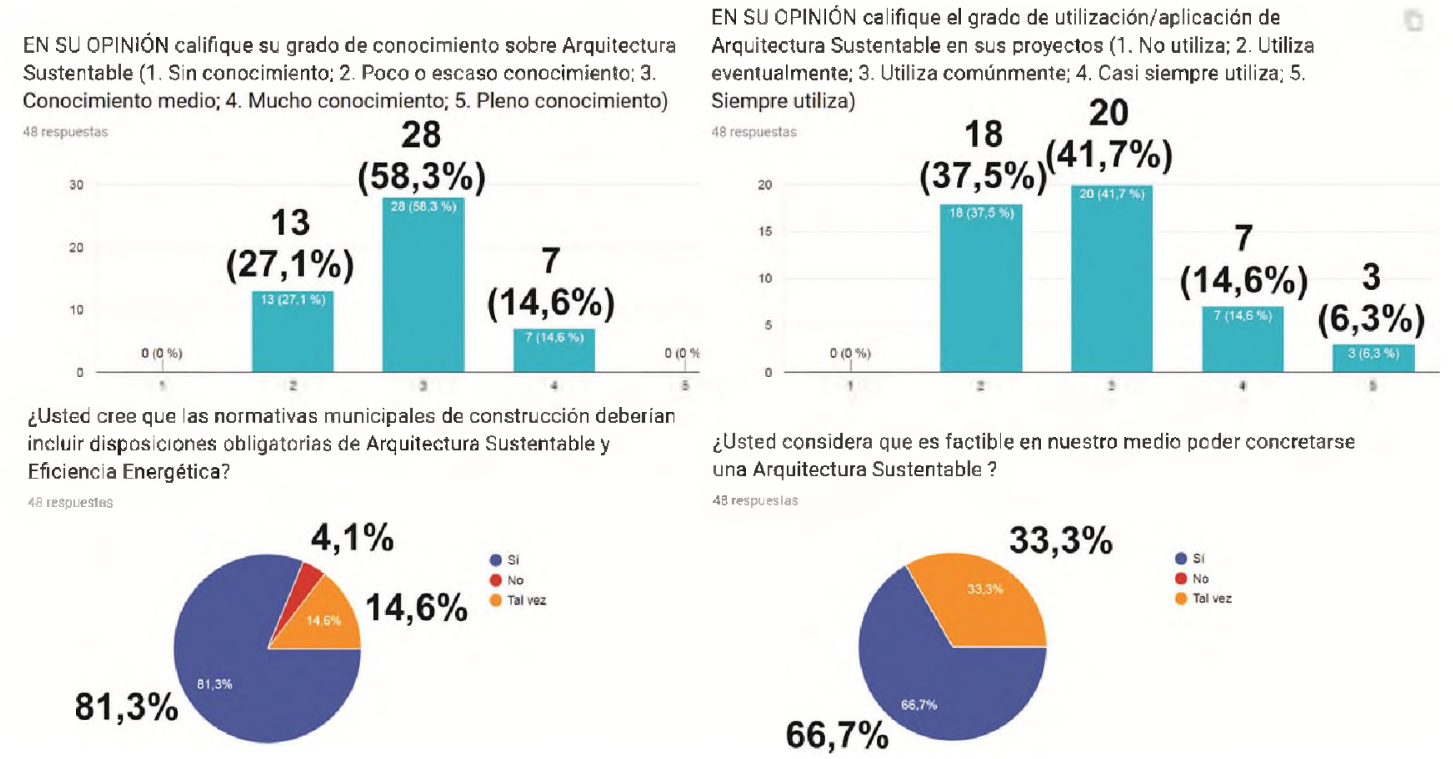

Figura 1 Resumen de resultados obtenidos.

${ }^{3}$ Obsérvese que la migración a Buenos Aires coincide con un contexto político y socio-económico adverso. 
acontezca en la construcción.

Encuesta: De los 48 encuestados, el $50 \%$ tiene entre 1 y 5 años de antigüedad en la profesión. E1 20,8\% de 6 a 10 años. E1 $10.4 \%$ de 10 a 20 años y el $18,8 \%$ más de 20 años. Del total de la muestra el 45,8\% trabaja en forma autónoma-independiente. $\mathrm{E} 135,4 \%$ en relación de dependencia. El $10,4 \%$ en sociedad y el $8,3 \%$ en otras formas.

\section{DISCUSIÓN:}

Al pensar en la necesaria inserción de la AS en la PC se cuestiona a los actores que la conforman para entender su comportamiento frente al posible cambio, y en especial, para entender "qué" cambios deben realizarse y "cómo" deben implementarse.

Es empíricamente comprobable y por tanto, descartable la posibilidad de que la PC genere por si sola una AS: "Puesto que el habitus es una capacidad infinita de engendrar, con total libertad (controlada), unos productos -pensamientos, percepciones, expresiones, acciones- que siempre tienen como límite las condiciones histórica y socialmente situadas de su producción, la libertad condicionada y condicional que él asegura está tan alejada de una creación de novedad imprevisible como de una simple reproducción mecánica de los condicionamientos iniciales." (Bourdieu, 1980: p.90).

Por otro lado, cabe pensar en los efectos positivos de la información y/o formación en AS de los actores estudiados (profesionales y constructores). El trabajo de campo realizado pone de manifiesto los conoci- mientos empíricamente aprendidos en el caso de los constructores, y la formación académica sumada a los saberes adquiridos en la práctica profesional por parte de los arquitectos. Aun así, los actores, informados en la temática tratada, no pueden vincular estrictamente estos conocimientos con la práctica. "Efectivamente, el peso particular de las experiencias primitivas resulta, en lo esencial, del hecho que el habitus tiende a asegurar su propia constancia y su propia defensa contra el cambio a través de la selección que él opera entre las informaciones nuevas, rechazando, en cada caso de exposición fortuita o forzada, las informaciones capaces de cuestionar la información acumulada y sobre todo favoreciendo la exposición a dichas informaciones." (Bourdieu, 1980: p.98). Resta solo pensar cómo reutilizar las informaciones y formaciones adquiridas por los actores para poder vincularlas a la PC.

\section{CONCLUSIONES:}

Se plantea un conflicto generacional entre la arquitectura contemporánea de nuestro medio y la arquitectura sustentable. No se oponen aquí estilos arquitectónicos ni desarrollos tecnológicos en el área de la construcción, sino habitus según modos de generación diferentes, que poseen definiciones diferentes de lo imposible, de lo posible y de lo probable, $y$ hacen que los unos experimenten como natural o razonable unas prácticas que los otros sienten como impensables o escandalosas, y a la inversa. El conflicto grafica porqué nos 
dedicamos a vender e instalar aires acondicionados en vez de acondicionar nuestras edificaciones (Bourdieu, 1980). En la búsqueda de los mecanismos que posibiliten la inserción eficiente de la AS en la PC, se permite inferir que la clave de estos no reside en los contratistas puesto que ellos se adaptarían a las nuevas maneras de construir. Por otro lado, los arquitectos manifiestan estar dispuestos a aprender y/o seguir sumando conocimientos sobre el tema y de hecho, creen factible la incorporación de la AS en los códigos de edificación, así como también, ven posible la materialización de una arquitectura sustentable en los tiempos venideros.

Se destaca las potencialidades, el amplio espectro de influencia del trabajo de los constructores en la provincia, y la visión optimista de los profesionales sobre la AS. Aun así, se desglosa la importancia de los dos actores estudiados en la búsqueda de la inserción de la AS, otorgándoles un rol de "acompañante" del posible cambio que deja las mayores responsabilidades en manos de la academia, los proveedores de materiales, los consumidores y por supuesto, los gobiernos locales. Cuál de estos últimos cuatro es el motor traccionador, cuales son los engranajes que simplemente tienen que acompañar el movimiento, son preguntas a resolver en futuros estudios. Mientras tanto, se infiere que podemos contar con los constructores y los profesionales para acompañar el proceso de inserción de la Arquitectura Sustentable.

\section{BIBLIOGRAFÍA:}

Bourdieu, P. (1980) El sentido práctico. Buenos Aires. Grupo editorial Siglo veintiuno. ISBN 978-987-1220-84-7

Gutierrez, A. (2005) Las prácticas sociales: una introducción a Pierre Bourdieu. Córdoba. Ferreyra Editor. ISBN 987-1110-20-0

ONU (1987) Nuestro futuro común. Comisión Mundial sobre el Medio Ambiente y el Desarrollo. 